\title{
Electrostatics of inter-Landau-level diodes
}

\author{
A. V. Khaetskii* \\ Faculty of Applied Physics and Delft Institute for Microelectronics and Submicrontechnology, Delft University of Technology, \\ Lorentzweg 1, 2628 CJ Delft, The Netherlands \\ V.I. Fal'ko ${ }^{\dagger}$ \\ Max-Planck-Institut für Festkörperforschung, Heisenbergstrasse 1, 70569 Stuttgart, Germany \\ G.E.W. Bauer \\ Faculty of Applied Physics and Delft Institute for Microelectronics and Submicrontechnology, Delft University of Technology, \\ Lorentzweg 1, 2628 CJ Delft, The Netherlands
}

(Received 10 December 1993; revised manuscript received 23 March 1994)

\begin{abstract}
We present exact solution of an electrostatic problem in terms of the potential distribution and density profile of an incompletely depleted two-dimensional electron gas in a heterostructure covered by a half-infinite gate. Using this solution, we calculate configurations of compressible and incompressible (insulating) strips that are formed in the presence of a quantizing magnetic field. We discuss the application of our results to transport experiments on the inter-Landau-level and spin diodes, as well as edge-magnetoplasmon propagation.
\end{abstract}

\section{INTRODUCTION}

A real two-dimensional (2D) electron system always contains macroscopic inhomogeneities created either by an external metallic gate, by etching, or by an external donor potential. The role of such macroscopic inhomogeneities increases significantly in the presence of a quantizing magnetic field. This is due to the fact that the chemical potential of a homogeneous two-dimensional electron gas (2DEG) in a magnetic field is not a continuous function of electron density $n$, but experiences a jump each time the density reaches the value at which an integer number of Landau levels is filled. As a result, the details of the screening are highly dependent on the filling factor $\nu=n / n_{L}\left(n_{L}=1 / 2 \pi \lambda^{2}, \lambda\right.$ being the magnetic length) and the inhomogeneous electronic system is split into compressible (metallic) and incompressible (insulating) regions. ${ }^{1-5}$

In experiments, the inhomogeneity is frequently created by applying a negative potential to a gate, the electron gas under the gate being partially depleted. In this case, far from the gate edge (on the right $x=\infty$, and left $x=-\infty$ ), we deal with half-infinite compressible (incompressible) regions separated by a transition region (strip). This transition region near the gate edge is the subject of the present study. Its structure plays a crucial role in the formation of electron transport across the inhomogeneity region (for instance, in the inter-Landau-level and spin diodes designed in the Corbino geometry ${ }^{6,7}$ ) and may be important for the quantitative description of the edge magnetoplasmons which can propagate along the border of two 2DEG's with different densities. ${ }^{8-11}$

\section{ELECTROSTATICS OF THE INCOMPLETELY DEPLETED 2DEG}

The structure we consider below is shown in Fig. 1(a). The inhomogeneity in the 2DEG is created by applying a negative voltage $-V_{g}$ to the metal half plane serving as a gate and lying on top of the heterostructure. The half space $z<0$ is occupied by a semiconductor with dielectric constant $\epsilon \gg 1$. The system is translationally invariant in the $y$ direction. The 2DEG plane is buried at a distance $d$ below the semiconductor surface. We assume that, at $V_{g}=0$, the electron concentration is uniform and equal to $n_{0},{ }^{12}$ and that at $V_{g} \neq 0$ the traditional concept of a capacitance is applicable, i.e., when solving the electrostatic problem, we assume the following boundary conditions: the electrostatic potential of the gate is $\varphi=-V_{g}$, and the electrostatic potential in the 2DEG plane is equal to zero. The boundary conditions at $z=0, x<0$ (uncovered surface of a heterostructure) are

$$
\left.\frac{d \varphi}{d z}\right|_{z=+0}=\left.\epsilon \frac{d \varphi}{d z}\right|_{z=-0},\left.\frac{d \varphi}{d x}\right|_{z=+0}=\left.\frac{d \varphi}{d x}\right|_{z=-0} .
$$

For the sake of simplicity and due to the fact that $\epsilon \gg 1$, we replace Eq. (1) by the simplified condition $d \varphi /\left.d z\right|_{z=-0}=0$ (for $x<0$ ). This makes it easier to apply methods of complex analysis and to obtain an exact analytical solution of the electrostatic problem. (In GaAs, $\epsilon \approx 13$, so that the accuracy of the above approximation is on the level of $10 \%$; below we will discuss the character of the solution obtained beyond this approximation.) 

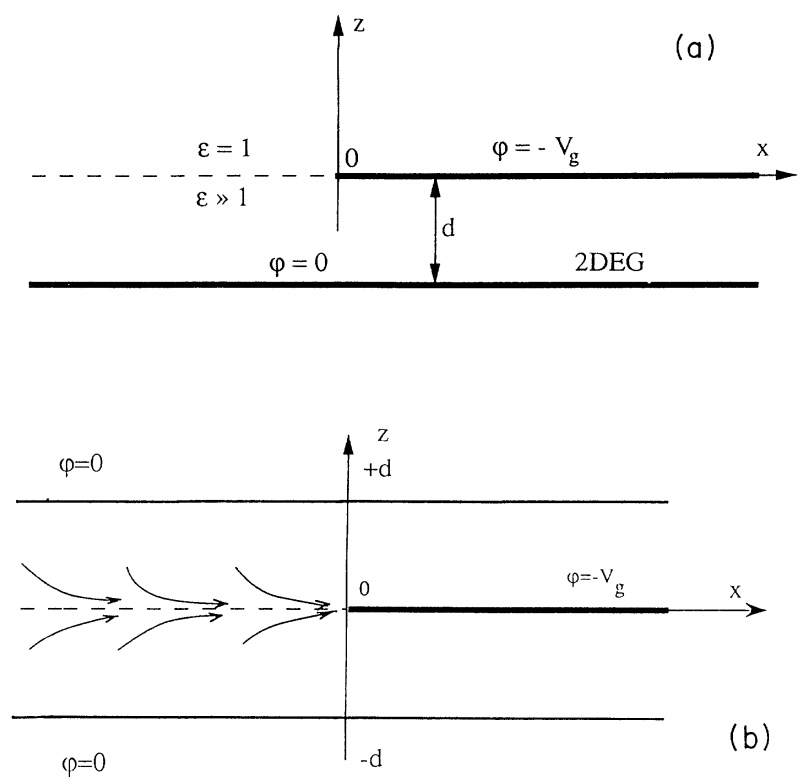

FIG. 1. (a) Geometry of the device considered. Half plane $z=0, x \geq 0$ is occupied by a metallic gate. (b) Plane of the complex variable $\xi=x+i z$. Curves with arrows show the direction of the electric field lines.

Consideration of the electrostatic potential alone is not sufficient for solving the problem of splitting of the electronic system into compressible and incompressible regions. Incompressibility follows from the fact that, at high magnetic fields, the spectrum of the 2DEG has gaps, and, at some filling factors $\nu$, the chemical potential $\mu(n)$ has discontinuities (jumps),

$$
\Delta \mu=\left\{\begin{array}{l}
\hbar \omega_{c} \text { for } \nu=2,4,6, \ldots, \\
g^{*} \mu_{B} H \text { for } \nu=1,3,5, \ldots,
\end{array}\right.
$$

where $H$ is the magnetic field, $\mu_{B}$ the Bohr magneton, $g^{\star}$ the effective $g$ factor, and $\omega_{c}=e H / m c$. The presence of each discontinuity in $\mu$ leads, in the case of a weakly inhomogeneous 2DEG, to the formation of incompressible strips of macroscopic width (much larger than the magnetic length). In these insulating regions the electron density is constant, $n=k n_{L}$, and the chemical potential $\zeta+e \varphi$ lies in the energy $\operatorname{gap}^{13}(\zeta$ is the electrochemical potential which is constant throughout the 2DEG). On the other hand, in the compressible regions (when the chemical potential $\mu$ is outside the gap) we have $\zeta=-e \varphi(x)+\mu(n(x))$, where $n(x)$ is the local electron density. ${ }^{13}$

This problem is appreciably simplified by the fact that $e V_{g} \gg \Delta \mu$. For this reason we can find a zero-order electron density distribution neglecting the internal energy $\mu$ of the 2DEG and then improve the solution using perturbation theory with respect to the small parameter $\mu / e V_{g}$. We stress that this procedure can give only small corrections for the value of the equilibrium electron density. For example, if $\nu<1$ the characteristic value of the internal energy is the exchange energy $e^{2} / \epsilon \lambda$. Therefore, for typical applied voltage and magnetic field we get for the correction $\delta n / n \simeq\left(e^{2} / \epsilon \lambda\right) / e V_{g} \leq 0.1$. However, near the spatial point where the filling factor is equal to an integer (and where an incompressible region is formed), the internal energy plays an important role, because it is the internal energy that determines the jump in the chemical potential. In these cases (for example, when calculating the width of an incompressible strip), the value of the internal energy should be taken into account explicitly (see Sec. III). Proceeding from the foregoing explanations, we first solve the purely electrostatic problem, when everywhere in the 2DEG plane $\mu(n) \equiv 0$, and find the electron density $n(x)$ for this case.

The change of the electron density $n_{0}-n(x)$ under the action of the voltage applied to the gate is found from

$$
\begin{array}{r}
\left.E_{z}\right|_{z=-d+0}=-\left.\frac{d \varphi}{d z}\right|_{z=-d+0}=\frac{4 \pi}{\epsilon} e\left[n_{0}-n(x)\right] \\
\left(\left.E_{z}\right|_{z=-d-0}=0\right) .
\end{array}
$$

The potential distribution $\varphi(x, z)$ is obtained from the solution of the Laplace equation $\nabla^{2} \varphi=0$ (there are no external charges outside the 2DEG plane) in the strip $-d \leq z \leq 0$ with the following boundary conditions:

$$
\varphi(x,-d)=0, \varphi(x \geq 0,0)=-V_{g},
$$

$$
\left.\frac{d \varphi}{d z}\right|_{z=-0}(x<0)=0 .
$$

This problem is equivalent to searching for the symmetric solution of the Laplace equation in a strip of width $2 d$ [see Fig. 1(b)] with the boundary conditions $\varphi(x, \pm d)=0$ and $\varphi(x>0, z=0)=-V_{g}$ (on the cut passing through the semi-infinite axis $x>0$ ). It is natural to make the conformal mapping

$$
U(\xi)=\exp (\pi \xi / d), \quad \xi=x+i z,
$$

which transforms the strip in Fig. 1(b) into the complex plane $U$ with cuts $\operatorname{Im} U=0, \operatorname{Re} U \leq 0(\varphi=0)$ and $\operatorname{Im} U=$ $0, \operatorname{Re} U \geq 1\left(\varphi=-V_{g}\right)$. In the complex plane $U$ the solution of the Laplace equation with the above boundary conditions is ${ }^{14}$

$$
\varphi(U)=-V_{g}\left\{1-\frac{1}{\pi} \operatorname{Im} \ln [2 U-1-2 \sqrt{U(U-1)}]\right\} .
$$

Now it is easy to obtain the solution $\varphi(x, z)$ of the initial electrostatic problem [see Eq. (3)], and using Eq. (2) to find the main approximation for the density profile in the system:

$$
n(x)=n_{0}-\frac{C V_{g}}{e} \frac{1}{\sqrt{1+\exp (-\pi x / d)}},
$$

where $C=\frac{\epsilon}{4 \pi d}$ is the capacitance per unit area and $n_{0}$ is the electron concentration at $V_{g}=0$ which coincides 
with the electron density far from the gate $(x \rightarrow-\infty)$. It should be noted that, due to the assumed incomplete depletion of the 2DEG under the gate, Eq. (5) differs from the corresponding result by Chklovskii et al.: ${ }^{4}$ the variation of the $2 \mathrm{D}$ density is localized within the width $\sim d$, whereas far from the gate edge the density variation is exponentially small. [The last statement, strictly speaking, is true only for the right side of the structure (under the gate); see the discussion below.]

As mentioned above, solution (5) was obtained in the lowest order in the small parameter $1 / \epsilon$. To understand the character of the corrected solution, we can take a look at the electrostatic problem similar to that we have already solved, but taking the dielectric constant to be the same in the whole space (at $z>0$ and $z<0$ ). Then we immediately come to the problem of a semiinfinite capacitor [to this end, Fig. 1(a) is symmetrically reflected with respect to plane $z=-d$, taking the semiplane $z=-2 d(x>0)$ at potential $\varphi=+V_{g}$. This problem can be solved exactly using the Schwarz-Christoffel transformation (see, e.g., the solution in Ref. 15). Then, calculating the normal derivative (with respect to $z$ ) of the potential at $z=-d$, we see that this quantity decays as $1 / x$ for large negative $x$ (to the left of the gate edge). Under the gate this solution coincides with the exponential function (5). Thus, at large negative $x$ the electron density has an asymptotic behaviour $\propto 1 / x$. The coefficient of proportionality in this dependence for a real experimental configuration [see Fig. 1(a)] can be found in the following way. At large (compared to $d$ ) negative $x$ the electrostatic potential of the boundary $z=0$ approximately equals zero with small corrections of the order of $V_{g}(d / x)$ (as follows from the consideration of equipotential lines). Thus, in order to calculate the $z$ component of the electric field at $z=+0$ (and large negative $x$ ) we should solve the Laplace equation in the half space $z>0$ with boundary conditions $\varphi(x \geq 0,0)=-V_{g}, \varphi(x \leq 0,0)=0$. The solution of this problem is just $\varphi=-V_{g}(1-\theta / \pi)$, where $\theta$ is a polar angle in the plane of the complex variable $\xi$. Calculating then the $z$ component of the electric field at $z=+0$ and using the first equality of Eq. (1) in order to calculate the corresponding quantity at $z=-d+0$, we finally get with the help of Eq. (2)

$$
n(x)=n_{0}+\frac{V_{g}}{4 \pi^{2} e x}
$$

Comparing Eqs. (5) and (6), we obtain that the asymptotic dependence given by Eq. (6) holds at $|x| \gg$ $d \ln \epsilon, x<0$. It should be noted that Eq. (6) is similar to the corresponding asymptotic formula in Ref. 4 where the case of a completely depleted 2DEG under the gate was considered. The only difference is that the second term in Eq. (6) contains an additional small factor $1 / \epsilon$ compared to the corresponding term in Ref. 4 . Thus, at large distances to the left of the gate edge, the characteristic scale of the potential experienced by the electrons is $V_{g} / \epsilon$ rather than $V_{g}$. As follows from the foregoing considerations, this is due to the incomplete depletion of the 2DEG under the gate [the electric field can penetrate into the 2DEG plane only from above; see Fig. 1(a)].

\section{INCOMPRESSIBLE STRIP FORMATION IN HIGH MAGNETIC FIELD}

\section{A. Formation of an incompressible strip between two compressible regions}

Now we take into account the influence of the internal energy by perturbation theory. ${ }^{4}$ As mentioned above, the chemical potential of the 2DEG in the quantizing magnetic field experiences jumps $\Delta \mu$ at integer values of the filling factor. This is equivalent to incompressibility of those regions where $\nu=1,2,3, \ldots$. Since $e V_{g}$ is much larger than the energy gap $\Delta \mu$, the magnetic field does not change the electrostatic density profile significantly. In the case of compressible regions on both sides of the gate edge [for instance, $\nu(-\infty)=5 / 2, \nu(+\infty)=3 / 2$ ], the only effect of the internal energy is the formation of a narrow strip of incompressible phase near point $x_{0}$, where the electron density corresponds to the filling factor $\nu=2$ :

$$
\frac{n\left(x_{0}\right)}{n_{L}}=2, \quad x_{0}=-\frac{d}{\pi} \ln \left[\left(\frac{n_{0}-n(+\infty)}{n_{0}-2 n_{L}}\right)^{2}-1\right] .
$$

Within this strip, the electron concentration is constant and equal to $2 n_{L}$ and the width $W_{\text {inc }}$ of the strip can be found following Chklovskii et al. ${ }^{4}$ as

$$
W_{\mathrm{inc}}^{2}=\frac{4 \epsilon \hbar \omega_{c}}{\left.\pi^{2} e^{2}(d n / d x)\right|_{x=x_{0}}} .
$$

The derivative $d n(x) / d x$ of function (5) is taken at point $x_{0}$ [see Eq. (7)], and Eq. (8) was derived for the limit of $W / d \ll 1$. This is indeed the case if the values of the filling factor at $x \rightarrow \pm \infty$ are not anomalously close to integer ones, since, according to Eqs. (5)-(8), the width of the incompressible strip is of the order of

$$
W_{\text {inc }} \sim d \sqrt{\frac{\hbar \omega_{c}}{e V_{g}}} .
$$

On the other hand, $W$ can be varied easily with gate voltage from the value Eq. (9) up to $W \sim d$.

\section{B. Formation of a compressible strip between two incompressible regions}

The experimental situation where regions of incompressible liquid exist on both sides of the gate edge (at $x \rightarrow \pm \infty$ ) is also frequently encountered, for instance, $\nu=2$ under the gate and $\nu=4$ far from the gate (here we neglect spin splittings). Let us consider the region under the gate. It should be noted that the integer value of the filling factor under the gate corresponds to an electron concentration at which the chemical potential of the 
2DEG $\mu(n)$ experiences a jump. However, owing to the infinite dimensions of the system, a certain value of the chemical potential at $x \rightarrow+\infty$ has some meaning. Finite temperature or weak disorder cause a large (but finite) screening radius $r_{s}$. Hence, at the scale $x \gg r_{s}$ the liquid is (weakly) compressible and the chemical potential is defined and pinned in the middle of the gap (see Fig. 2). Yet, at the scale $x \ll r_{s}$ (near the gate edge) the local value of the chemical potential in the incompressible liquid is not defined. The adequate criterion for the determination of the boundary $x_{R}$ between the compressible and incompressible regions is

$$
\delta \varphi=\varphi(+\infty)-\varphi\left(x_{R}\right)=-\left(\frac{\Delta \mu_{R}}{2 e}\right),
$$

where $\Delta \mu_{R}$ is the magnitude of the jump of the chemical potential at an integer value of the filling factor $\nu$ under the gate (see Fig. 2) (in the example considered, $\left.\Delta \mu_{R}=\hbar \omega_{c}\right)$. To find the boundary of the compressible region $\left[x_{R}\right.$ from Eq. (10)], we should determine the distribution of the electrostatic potential in our system by taking into account the real charge distribution. $n(x)$ in this case differs from that in Eq. (5) by the fact that the electron concentration is constant at $x>x_{R}$ due to the incompressibility of the electron liquid. Moreover, the presence of a jump in the chemical potential gives rise to a jump in the capacitance of the semi-infinite capacitor at this value of $\nu$. Therefore, the value of $V_{g}$ related to the classical capacitance should be slightly corrected by $\Delta \mu_{R} / 2 e$. Now we can use the solution (5), but with a corrected value of $V_{g}$. The value of the concentration $\tilde{n}(x)$ so calculated will be lower at $x \rightarrow \infty$ as compared to $2 n_{L}$. Proceeding from the relationship $\delta n / n \sim \delta \varphi / V_{g}$, we can find the edge of the incompressible region $x_{R}$ from the condition

$$
\frac{\tilde{n}\left(x_{R}\right)-\tilde{n}(+\infty)}{2 n_{L}} \simeq \frac{\Delta \mu_{R}}{2 e V_{g}} .
$$

Since $\Delta \mu_{R} / e V_{g} \ll 1$, in Eq. (11) we can use the expression for $\tilde{n}(x)$ which is valid for large $x(x \gg d)$. As a

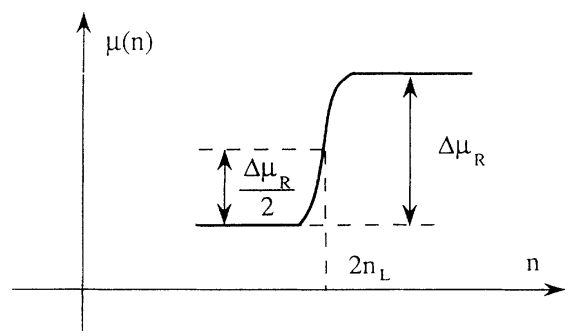

FIG. 2. Dependence of the chemical potential of a 2DEG in a magnetic field on electron concentration near the value corresponding to an integer filling factor. $\Delta \mu_{R}$ is the value of the jump of the chemical potential at an integer filling factor under the gate. Analogously, $\Delta \mu_{L}$ is the value for the left edge of the device. At the far right edge of the device, the chemical potential is pinned and has a value corresponding to $n=2 n_{L}$. The screening radius is $r_{s}=\left(\epsilon / e^{2}\right) d \mu / d n$, where the derivative is calculated at $n=2 n_{L}$. result, we get

$$
x_{R}=+\frac{d}{\pi}\left[\ln \left(\frac{e V_{g}}{\Delta \mu_{R}}\right)+a\right] .
$$

These arguments are valid only in the logarithmic approximation when $\ln \left(e V_{g} / \Delta \mu_{R}\right) \gg 1$ and, hence, the specific value of $a \sim 1$ in Eq. (12) is not important (the answer obtained is true when $x_{R}<r_{s}$ ). [Using the Schwarz-Christoffel transformation, it is possible to prove Eq. (12) rigorously and find the value of the quantity $a=\ln 2$ (Ref. 16). The corresponding solution is cumbersome and we do not present it here.]

Concerning the left side of the structure, the incompressible region cannot occupy the whole semiplane to the left from some point $x_{L}$. This is clear from the following simple consideration. Since at $x=-\infty$ the electron liquid is neutral and the electron concentration is constant in the incompressible region, there exists local neutrality up to point $x_{L}$ (electron concentration equals the background positive charge density). To the right from this point the electrostatic potential is constant (a compressible, metallike, strip). But near the edge of the metal plane the electric field has a square root singularity, which means that the electron concentration is not a continuous function of the coordinates and the solution we tried to construct cannot actually be realized. This difficulty exists even in the lowest order in parameter $1 / \epsilon$ [when solution (5) holds] and is related to the absence of the gate on the left side of the structure. The details of incompressible strip formation in this region depend strongly on the value of the quantity $\Delta \nu(-\infty) \equiv \nu(-\infty)-4$ (for the case considered). We discuss only the case when this quantity, being positive, tends to zero and $\Delta \nu(-\infty) \ll(\epsilon \ln \epsilon)^{-1}$. It means that the point $x_{0}$ where the filling factor equals 4 lies to the left of the crossover point $\tilde{x} \sim d \ln \epsilon$ and the variation of electron concentration is described by formula (6). Thus we have for $x_{0}$

$$
x_{0}=-\frac{V_{g}}{4 \pi^{2} e n_{L} \Delta \nu(-\infty)} .
$$

As has already been mentioned, the characteristic scale of the electrostatic potential experienced by electrons in this region is $V_{g} / \epsilon$. Therefore, if $\Delta \mu_{L} \ll V_{g} / \epsilon$, the incompressible strip is formed with a width much smaller than the distance $x_{0}$ between the strip and the gate edge (to describe this situation we may use the formulas of Ref. 4). But if $\Delta \mu_{L} \simeq V_{g} / \epsilon$ (the case which could have been realized in experiments $\left.{ }^{6,7}\right)$, then the width of the incompressible strip formed is of the order of $x_{0}$ (i.e., relatively wide). Hence, this case cannot be considered using a perturbative approach. ${ }^{4}$ We were not able to obtain the exact solution of the corresponding electrostatic problem. But it is clear that this solution exists, and for this parameter range the width of the incompressible strip formed is of the order of $x_{0}$, and the distance between the gate edge and the nearest edge of the strip $x_{L}$ is slightly smaller, but of the same order. Of course, the physical pattern described is realized only if $\left|x_{0}\right| \ll r_{s}$ (for the definition of $r_{s}$ see the caption of Fig. 2, and the corre- 
sponding derivative $d \mu / d n$ is calculated at $\left.n=4 n_{L}\right)$.

Everywhere above, when referring to the incompressible state on the left side of the structure [with $\nu(-\infty)=$ 4], we meant real incompressibility (in particular, the electron concentration is strictly constant). But we should bear in mind that because of the abrupt variation of the electron concentration near the gate edge [see Eq. (5)] the electron concentration becomes very close to $4 n_{L}$ at a finite distance from the gate edge. For example, at $\tilde{x} \simeq d \ln \epsilon$ [where the crossover between the dependences described by Eqs. (5) and (6) occurs] the deviation of concentration from $4 n_{L} \approx n_{0}$ is of the order of $n_{0} /(\epsilon \ln \epsilon)$. Therefore, from a practical point of view, the electron liquid in the region to the left of some spatial point (e.g., $x \leq \tilde{x}$ ) will behave as an incompressible one (with very poor screening properties).

\section{DISCUSSION}

The above calculated value of $W_{\text {inc }}$ [see Eq. (8)] can be measured experimentally, ${ }^{6}$ since the incompressible region is insulating and at low temperatures the current in the transverse direction is caused by electron tunneling. At a high magnetic field the tunneling is mainly determined by the effective magnetic barrier. When $W_{\text {inc }} \gg \lambda$, the tunneling probability is exponentially small. Hence, the value of $W_{\text {inc }}$ can be derived from experiments with logarithmic accuracy when a formula for the conductance is available. When deriving such a formula, one should bear in mind that tunneling in a transverse magnetic field has to be accompanied by transfer of momentum in the direction perpendicular to the current. We assume that such a process may be stimulated by a defect located in the incompressible region. Most efficient are the impurities located near the center of the incompressible strip. ${ }^{17}$ For the conductance we obtain

$$
G \propto \exp \left(-\frac{W_{\text {inc }}^{2}}{2 \lambda^{2}}\right) .
$$

The foregoing reasons can be applied to the case when the jump of the chemical potential occurs at $\nu=1$. The incompressible state corresponding to $\nu=1$ is assumed to be spin polarized and the elementary charged excitation is an electron with the opposite orientation of spin. ${ }^{18}$ In the independent particle picture the jump $\Delta \mu$ is determined by the bare Zeeman splitting in the 2DEG: $\Delta \mu=g_{0} \mu_{B} H$. On the other hand, the theory of exchange effects in a homogeneous $2 \mathrm{D}$ electron system in a magnetic field ${ }^{19}$ yields a much greater value, $\Delta \mu \sim e^{2} / \epsilon \lambda$, which is the characteristic Coulomb energy in the system. The latter result, which, strictly speaking, is valid for the homogeneous system, seems to be applicable to the case in question when $d \gg W \gg \lambda$. This can be experimentally verified with the use of Eqs. (5) and (8), where in Eq. (8) $\Delta \mu$ should be substituted for $\hbar \omega_{c}$. We tentatively interpret the experimental data ${ }^{7,20}$ as evidence against the equality $\Delta \mu=g_{0} \mu_{B} H$ : the experiment ${ }^{7}$ revealed a pure activation behavior of the zero-bias conductance at low temperatures, ${ }^{20}$ whereas the substitution of bare Zeeman splitting into Eq. (8) brings about an appreciable value of the tunneling conductance. The anomalously small value of the zero-bias tunneling conductance in experiment ${ }^{7}$ is caused by the large value of $d \approx 6000 \AA$. On the other hand, as seen from Eq. (8), $W_{\text {inc }} \propto \sqrt{d}$. Therefore, for structures with a much smaller value of $d$, it should be possible to reach a regime where the tunneling conductance is significantly high such that $\Delta \mu$ can be determined with the aid of Eqs. (5), (8), and (14).

The propagation of edge magnetoplasmons along the border of a 2DEG (Refs. 8-11) is another physical effect which is sensitive to the structure of the transitional region. The experimental conditions for observation of edge magnetoplasma excitations are mainly restricted to the integer filling factors in both (left and right) semiinfinite planes, which provide minimal friction and slowest relaxations. Therefore, this deals with the second of the examples considered above (Sec. III B ). The width of the compressible region, in such a case, determines the width of a strip where the magnetoplasmon charge can be accumulated, and therefore is an important quantity determining the dispersion of this mode. Calculation of this dispersion law is a separate problem to be solved. However, some qualitative features of this dispersion law can be understood from the analogy of this problem with that of edge-magnetoplasmon (EMP) propagation in the $2 \mathrm{D}$ electron system on the surface of liquid helium. ${ }^{9}$ In the latter case the geometry of the system is similar to that considered here because there are metallic plates near the $2 \mathrm{D}$ electron layer. That is why we can expect softening of the EMP frequency for $q d \ll 1$ ( $q$ is the wave vector of the EMP along the border) (see Ref. 9). In particular, since in the case considered here the characteristic spatial scale of the variation of the electron density is of the order of the distance to the metallic gate, it is highly probable that this dispersion law will not contain the usual logarithmic factor $\ln (1 / q b)$ in the limit $q \rightarrow 0$. Moreover, our solutions of the electrostatic problem can be applied to the calculation of a multiple-strip structure of the inter-Landau-level diode. When the distance between a gate and the 2DEG is large enough, the density distribution $\nu(x \rightarrow-\infty)=4$ and $\nu(x \rightarrow \infty)=2$ can be associated with the existence of an additional incompressible strip (with $\nu=3$ ) inside the compressible one, which produces an additional (as compared to the conventional edge magnetoplasmon) mode in the excitation spectrum of this system. ${ }^{10}$

\section{ACKNOWLEDGMENTS}

We thank D. Chklovslkii, R. Gerhardts, I. Grodnensky, Yu. Nazarov, and B. Shklovskii for useful discussions and, especially, B. Kane who has made available for us some unpublished information related to his earlier works. ${ }^{6,7}$ This work is a part of the research program of the "Stichting voor Fundamenteel Onderzoek der Materie (FOM)." Two of us (A.V.Kh. and G.E.W.B.) acknowledge financial support from the "Nederlandse Organisatie voor Wetenschappelijk Onderzoek (NWO)." One of us (V.F.) acknowledges financial support from the Alexander von Humboldt Foundation. 
* Permanent address: Institute of Microelectronics Technology, Russian Academy of Sciences, 142432 Chernogolovka, Moscow District, Russia.

' On leave from Theory Department, Institute of Solid State Physics, Russian Academy of Sciences, 142432, Chernogolovka, Moscow District, Russia.

${ }^{1}$ C.W.J. Beenakker, Phys. Rev. Lett. 64, 216 (1990); A.M. Chang, Solid State Commun. 74, 871 (1990).

${ }^{2}$ A.L. Efros, Solid State Commun. 67, 1019 (1988); Phys. Rev. B 45, 11354 (1992).

${ }^{3}$ P.L. McEuen, E.B. Foxman, J. Kinaret, U. Meirav, M.A. Kastner, N.S. Wingreen, and S.J. Wind, Phys. Rev. B 45, 11419 (1992).

${ }^{4}$ D.B. Chklovskii, B.I. Shklovskii, and L.I. Glazman, Phys. Rev. B 46, 4026 (1992).

${ }^{5}$ D.B. Chklovskii, K.A. Matveev, and B.I. Shklovskii, Phys. Rev. B 47, 12605 (1993).

${ }^{6}$ B.E. Kane, D.C. Tsui, and G. Weimann, Phys. Rev. Lett. 61, 1123 (1988).

${ }^{7}$ B.E. Kane, L.N. Pfeiffer, and K.W. West, Phys. Rev. B 46, 7264 (1992).

${ }^{8}$ S.A. Mikhailov and V.A. Volkov, J. Phys. Condens. Matter 4, 6523 (1992).
${ }^{9}$ V.A. Volkov and S.A. Mikhailov, Zh. Eksp. Teor. Fiz. 94, 217 (1988) [Sov. Phys. JETP 67, 1639 (1988)].

${ }^{10}$ I. Grodnensky (unpublished).

${ }^{11}$ V.I. Talyanskii et al., J. Phys. Condens. Matter 5, 7643 (1993).

12 That is, $V_{g}=0$ is calibrated in such a way that it includes the Schottky barrier at the metal-semiconductor surface.

${ }^{13}$ B.I. Halperin, Helv. Phys. Acta 56, 75 (1983).

${ }^{14}$ J.C. Maxwell, $A$ Treatise on Electricity and Magnetism (Clarendon, Oxford, 1892).

${ }^{15}$ P.M. Morse and H. Feshbach, Methods of Theoretical Physics (McGraw-Hill, New York, 1953), Part 2.

${ }^{16}$ A. Khaetskii (unpublished).

${ }^{17}$ B.I. Shklovskii and A.L. Efros, Zh. Eksp. Teor. Fiz. 84, 811 (1983) [Sov. Phys. JETP 57, 470 (1983)].

${ }^{18}$ Yu. Bychkov, S.V. Iordanskii, and G.M. Eliashberg, Pis'ma Zh. Eksp. Teor. Fiz. 33, 152 (1981) [JETP Lett. 33, 143 (1981)]; C. Kallin and B.I. Halperin, Phys. Rev. B 30, 5655 (1984).

19 J.F. Janak, Phys. Rev. 178, 1416 (1969); T. Ando and Y. Uemura, J. Phys. Soc. Jpn. 37, 1044 (1978).

${ }^{20} \mathrm{~B}$. Kane (private communication). 